\title{
Pharmacotherapeutic aspects of treating knee osteoarthritis with glucosamine sulfate
}

\author{
Steven Simoens*, Gert Laekeman
}

Research Centre for Pharmaceutical Care and Pharmaco-economics, Katholieke Universiteit Leuven, Onderwijs en Navorsing, Leuven, Belgium; *Corresponding Author: steven.simoens@pharm.kuleuven.be

Received 5 February 2010; revised 11 March 2010; accepted 15 March 2010.

\begin{abstract}
Glucosamine sulfate is a natural constituent of cartilage and is used in the treatment of knee osteoarthritis. The aim of this study is to provide a short but comprehensive pharmacotherapeutic update on treating knee osteoarthritis with glucosamine sulfate. A literature search was conducted of PubMed, Centre for Reviews and Dissemination databases, Cochrane Reviews and EconLit up to January 2010. The literature review indicated that the mechanism of action of glucosamine sulfate is based on hypothesis, but its treatment effects in knee osteoarthritis are symptomatic. With steady-state peak concentrations at the $1,500 \mathrm{mg}$ dosage in the range of $10 \mu \mathrm{M}$, it is estimated that only $2 \%$ of glucosamine is incorporated in the cartilage. A once-daily dosage of $1,500 \mathrm{mg}$ of glucosamine sulfate is licensed for the treatment of symptomatic osteoarthritis and has been shown to reduce pain, improve function and exhibit similar safety to placebo. Glucosamine sulfate is likely to be a cost-effective treatment of knee osteoarthritis. In conclusion, a once-daily dosage of $1,500 \mathrm{mg}$ of glucosamine sulfate is likely to be a safe, effective and cost-effective treatment of knee osteoarthritis as compared to placebo.
\end{abstract}

Keywords: Pharmacotherapy; Glucosamine Sulfate, Knee Osteoarthritis; Pharmacokinetics; Indication; Safety; Effectiveness;

Cost-Effectiveness

\section{INTRODUCTION}

Knee osteoarthritis is associated with significant morbidity, impaired quality of life, and substantial healthcare costs [1]. Pharmacological treatment of knee osteoarthri- tis includes analgesics, non-steroidal anti-inflammatory drugs (NSAIDs), corticosteroids and opiods, and glucosamine sulfate. Glucosamine sulfate is a natural substance that is a constituent of cartilage. Glucosamine sulfate is sold as a dietary supplement in the United States, but is registered as a medicine throughout much of Europe.

To date, there is uncertainty over various pharmacotherapeutic aspects of treating knee osteoarthritis with glucosamine sulfate. Therefore, the aim of this study is to provide a short but comprehensive pharmacotherapeutic update on treating knee osteoarthritis with glucosamine sulfate.

\section{METHOD}

\subsection{Search Strategy}

A literature search was conducted of PubMed, Centre for Reviews and Dissemination databases, Cochrane Database of Systematic Reviews and EconLit up to January 2010. Additionally, the bibliography of included studies was checked for other relevant studies. Search terms included "knee osteoarthritis", "knee joint”, “glucosamine sulfate”, "pharmacokinetics”, "bioavailability”, "indications”, “dosage”, "safety”, “effectiveness”, "costeffectiveness", "economic evaluation” alone and in combination with each other.

The literature search included articles published in peer-reviewed journals and congress abstracts. Also, the researchers contacted manufacturers of glucosamine sulfate for any unpublished studies. The review was limited to studies published in English for practical reasons.

\subsection{Inclusion and Exclusion Criteria}

The review was limited to the use of glucosamine sulfate in its indication of knee osteoarthritis.

To explore the pharmacotherapy of glucosamine sulfate from a multidisciplinary perspective, the literature review assessed the following aspects: pharmacokinetics, 
indications and dosage, safety and effectiveness, and cost-effectiveness. Evidence about cost-effectiveness was derived from economic evaluations. An economic evaluation was defined as a study comparing glucosamine sulfate with an alternative treatment in terms of both costs and consequences.

\section{RESULTS}

\subsection{Search Results}

The results of the literature search are displayed in Figure 1. The review summarized three studies on pharmacokinetics, a Cochrane literature review on safety and effectiveness, and three economic evaluations of glucosamine sulfate in knee osteoarthritis.

\subsection{Pharmacokinetics}

A literature review analysed the absorption, distribution, metabolism and excretion of different administration forms of glucosamine sulfate [2]. The absolute bioavailability of oral glucosamine sulfate amounted to $44 \%$. The fecal excretion was $11.3 \%$ of the dosage, indicating that at least $88.7 \%$ of the dosage was absorbed through the gastrointestinal tract. With an absolute bioavailability of $44 \%$ calculated after oral 14C-glucosamine, a firstpass effect can be hypothesized.

A study examined the dosage-proportionality of glucosamine sulfate in 12 healthy volunteers [3]. The pharmacokinetics were linear in the $750 \mathrm{mg}$ and $1,500 \mathrm{mg}$ dosages, but not at 3,000 mg, where the plasma concentration-time profiles were less than expected based on dosage-proportionality. More recent research showed that glucosamine concentrations in plasma and synovial fluid were correlated [4]. There remains controversy over whether steady-state peak concentrations at the $1,500 \mathrm{mg}$ dosage were in line with those found to be effective in selected in vitro studies.

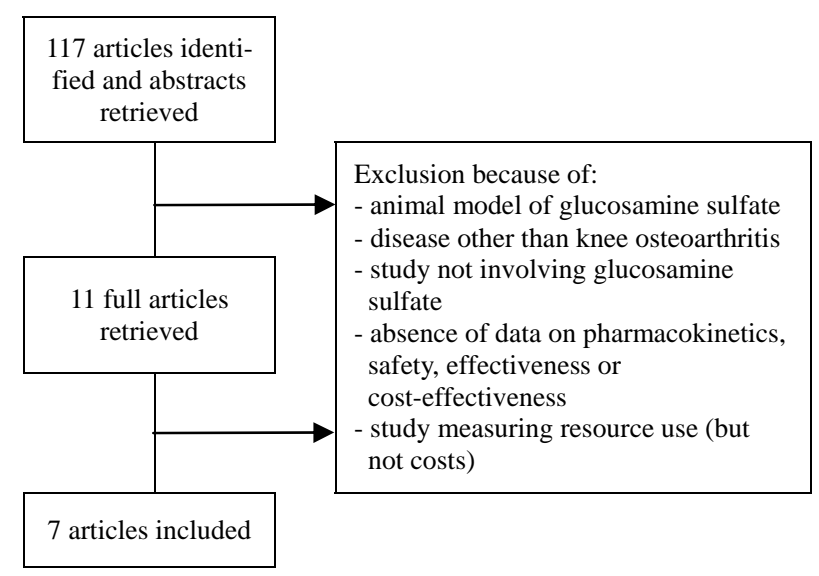

Figure 1. Flow chart of literature search.

\subsection{Indications and Dosage}

The indication of glucosamine sulfate in Europe is limited to the symptomatic treatment of knee osteoarthritis. An expansion of indication to osteoarthritis of the hip, spine, hands and other locations was refused, as a result of which some companies have refused to register their preparation as a medicine. Glucosamine sulfate can be used alone or in combination with NSAIDs. The licensed dosage is $1,500 \mathrm{mg}$, once daily. Symptom relief may not be experienced until after several weeks of treatment, and optimal effects on joint mobility have been observed after 12 weeks of administration.

\subsection{Safety and Effectiveness}

A Cochrane review assessed randomized controlled trials (RCTs) evaluating the effectiveness and toxicity of glucosamine in osteoarthritis up to the end of 2008 [1]. Of the 25 studies included in the review, 20 RCTs focused on the knee exclusively and five RCTs examined osteoarthritis at other or multiple sites. All studies were double-blinded randomized parallel-group trials, enrolling a total of 4,963 patients (mean age of 60.7 years, $69 \%$ were female). Fourteen RCTs had affiliations with Rotta Pharmaceuticals (an Italian manufacturer of glucosamine sulfate).

A pooled analysis of the relevant RCTs showed that, as compared to baseline, glucosamine sulfate taken orally in amounts of $1,500 \mathrm{mg} /$ day produced an increase in the total score of the Lequesne Index (a multidimensional index measuring knee pain or discomfort, activities of daily living and maximum distance walked) of $11 \%$ (standardized mean difference of -0.47 ; $95 \%$ confidence interval: -0.82 to -0.12 ). A subgroup analysis of the Rotta preparation showed significant benefit over placebo in terms of pain (standardized mean difference of -1.11 ; $95 \%$ confidence interval: -1.66 to -0.57 ) and in terms of the total score of the Lequesne Index (standardized mean difference of $-0.47 ; 95 \%$ confidence interval: -0.82 to -0.12 ). Studies using a non-Rotta preparation failed to show benefit over placebo in terms of pain and function as measured by the Western Ontario and McMaster Universities Index (a disease-specific index measuring pain, stiffness and function). Two RCTs suggested that the Rotta preparation of glucosamine sulfate may slow radiological progression of knee osteoarthritis over a three-year period (average difference of 0.32 ; $95 \%$ confidence interval: 0.05 to 0.58 ).

Glucosamine therapy exhibited a safety profile similar to placebo in terms of the number of patients with side-effects (relative risk ratio of 0.99 ; $95 \%$ confidence interval: 0.91 to 1.07 ) and significantly better than NSAIDs (relative risk ratio of 0.29 ; $95 \%$ confidence interval: 0.19 to 0.44 ). 


\subsection{Cost-Effectiveness}

A Belgian economic evaluation indicated that treatment of knee osteoarthritis with glucosamine sulfate for at least one year and up to three years was associated with a lower incidence of total knee replacement over an observation period of eight years [5]. Costs of glucosamine sulfate treatment were offset by lower hospitalization costs, resulting in overall savings. Total costs per patient amounted to $€ 1,103$ in the placebo group and $€ 901$ in the glucosamine sulfate group. In other words, treatment of knee osteoarthritis with glucosamine sulfate for up to three years is associated with savings of $€ 202$ per patient during the overall observation period of eight years (i.e. up to three years of treatment and five years of follow-up). This study indicated that glucosamine sulfate treatment was more effective and less expensive than placebo in knee osteoarthritis. If costs of other osteoarthritis medicines and other health care resource use are taken into account, the cost-effectiveness of glucosamine sulfate would even be more favourable.

The National Institute for Health and Clinical Excellence in England and Wales compared the likely costeffectiveness of selected treatments for knee osteoarthritis [6]. This study did not provide a full economic evaluation, but presented UK healthcare costs alongside the effectiveness of each treatment option. The scope of costs was limited to treatment costs (such as medicine costs), but did not include other healthcare costs such as adverse event costs or decreased use of other medical resources. The results suggested that glucosamine sulfate $(1,500 \mathrm{mg} /$ day) is likely to be cost-effective as compared with placebo.

A recent US analysis explored the cost-effectiveness of glucosamine sulfate treatment versus placebo in a hypothetical cohort of 100,000 patients who were 65 years old and had knee osteoarthritis for five years [7]. A hybrid Markov-decision tree model was constructed using published effectiveness data and US fee schedules. Treatment with a daily dosage of $1,500 \mathrm{mg}$ of glucosamine sulfate turned out to be more effective and less expensive than placebo by delaying time to joint replacement surgery. However, cost-effectiveness results were sensitive to changes in several input parameters and the authors recommended additional research to inform decision making.

\section{CONCLUSIONS}

This study provided a pharmacotherapeutic update on treating knee osteoarthritis with glucosamine sulfate. Additionally, an economic evaluation explored the costeffectiveness of treating knee osteoarthritis with glucosamine sulfate in Belgium. The main messages can be summarized as follows. The mechanism of action of glucosamine sulfate is based on hypothesis, but its treatment effects in knee osteoarthritis are symptomatic. A once-daily dosage of $1,500 \mathrm{mg}$ of glucosamine sulfate has been licensed for the symptomatic treatment of knee osteoarthritis. Glucosamine sulfate has been shown to reduce pain and improve function of patients with knee osteoarthritis. Glucosamine sulfate therapy exhibits a safety profile similar to placebo and significantly better than NSAIDs. Finally, glucosamine sulfate is likely to be a cost-effective treatment of knee osteoarthritis.

\section{ACKNOWLEDGEMENTS}

No funding was provided to support this study. The authors have no conflicts of interest that are relevant to the content of this manuscript.

\section{REFERENCES}

[1] Towheed, T., Maxwell, L., Anastassiades, T.P., Shea, B., Houpt, J.B., Welch, V., Hochberg, M.C. and Wells, G.A. (2005) Glucosamine therapy for treating osteoarthritis. Cochrane Database of Systematic Reviews, 18(2).

[2] Setnikar, I. and Rovati, L.C. (2001) Absorption, distribution, metabolism and excretion of glucosamine sulfate. A review. Arzneimittelforschung, 51(9), 699-725.

[3] Persiani, S., Roda, E., Rovati, L.C., Locatelli, M., Giacovelli, G. and Roda, A. (2005) Glucosamine oral bioavailability and plasma pharmacokinetics after increasing doses of crystalline glucosamine sulfate in man. Osteoarthritis and Cartilage, 13(12), 1041-1049.

[4] Persiani, S., Rotini, R., Trisolino, G., Rovati, L.C., Locatelli, M., Paganini, D., Antonioli, D. and Roda, A. (2007) Synovial and plasma glucosamine concentrations in osteoarthritic patients following oral crystalline glucosamine sulfate at therapeutic dose. Osteoarthritis and Cartilage, 15(7), 764-772.

[5] Simoens, S. (2009) Economic evaluation of glucosamine sulphate treatment in knee osteoarthritis. ISPOR's 12th Annual European Congress, Paris.

[6] National Institute for Health and Clinical Excellence. (2009) Osteoarthritis: The care and management of osteoarthritis in adults: NICE clinical guideline 59. http://www.nice.org.uk/nicemedia/pdf/CG59NICEguideli ne.pdf

[7] Ku, L.J.E. and Biddle, A. (2009) Cost-effectiveness of glucosamine sulfate treatment among elderly knee osteoarthritis patients. $7^{\text {th }}$ World IHEA Congress on Health Economics, Beijing. 